\title{
AN OPTIMAL TSALLIS ENTROPY BASED SEGMENTATION WITH DEEP EXTREME LEARNING MACHINE FOR MEDICAL IMAGE CLASSIFICATION USING MRI IMAGES
}

\author{
Deeparani K \\ Research Scholar, Department of Computer Science, \\ Bharathiar University, Coimbatore-641 046, TN, India \\ deeparanikannan@gmail.com \\ Sudhakar P \\ Associate Professor, Department of Computer Science, \\ Annamalai University, Chidambaram-TN, India \\ kar.sudha@gmail.com
}

\begin{abstract}
Medical image classification becomes an essential process in the healthcare sector for decision making using medicinal images. At the same time, brain tumor (BT) is considered the deadliest disease over the globe and it affects the brain tissues. So, it is needed to diagnose BT at the preliminary stages to enhance the survival rate of the patients. The goal of this paper is to derive an effective segmentation and classification technique for BT diagnosis. This paper designs an optimal Tsallis entropy based segmentation with deep extreme learning machine (OTSE-DELM) model for medical image classification using MRI images. The presented model involves multi-level thresholding based image segmentation using Tsallis entropy and the optimal threshold values are chosen by black widow optimization (BWO) algorithm. Besides, the presented model encompasses directional local extrema patterns (DLEP) based feature extractor. In addition, the OTSE-DELM model involves DELM based classification model to allocate the proper class labels of the test MRI images. A wide range of simulations was performed to showcase the betterment of the OTSE-DELM model and the outcomes are investigated under distinct dimensions. The experimental values make sure that the OTSE-DELM model is found to be superior to other methods by providing an increased sensitivity of $98.34 \%$, specificity of $98.98 \%$, and accuracy of $98.76 \%$.
\end{abstract}

Keywords: Brain tumor, image segmentation, image classification, deep learning, multi-level thresholding.

\section{Introduction}

Gliomas is a major kind of brain tumor (BT) that originates from the glial cells and about $70 \%$ of grownups are affected by this disease. The tumor cells exhibit faster development and it is classified into two types namely low grade (oligodendroglioma) and high grade (glioblastoma) [1]. If the tumors are identified at the primary level, it is less deadly over the advanced level that minimizes the patient's endurance rate to $<2$ years. Chemotherapy, therapy, surgical treatments, or their integration is provided to treat the tumor patients. Owing to the high multiplanar magnetic resonance (MR) resolution, magnetic resonance imaging (MRI) is commonly employed to detect gliomas.

Segmentation of MRI is an important process of monitoring the differences in the tumor sizes. It acts as a significant part in the radiotherapy/surgical planning to segregate the infected and normal brain regions. At the present time, the manual segmentation outcome is highly dominated in the medical routine where the radiologist necessitates more time.

In addition, the complicated glioma features and delicate differences among the MRI examination create inevitable issues to detect reliability by visually examining the MRI using the expert radiologist. Automated BT identification models are found to be effective and eloquent. Segmentation techniques for BT by the use of 2D slices of MRI undergo partition into distinct class types namely threshold, region based and pixel-based classification. Threshold methods do not offer $<1$ threshold for classifying the target voxel segmentation by the intensity levels. The second Sobel filtering is applied for outlining the edges of voxels. Afterward, the pixel values of all the voxels undergo comparison with threshold condition and the pixel values are allocated to the neighboring areas for refining the outcome. Region based models perform voxel isolation manually into limited districts [2]. It is a traditional model where an individual seed is planted in all districts and every voxel resemblance with the 
nearby seeds is determined. An extended version is developed to reduce the partial volume result and identifies refined edges of voxels by computing the gradient details. The adaptive region growing models are employed for accurately segmenting the tumor regions. Pixel oriented models make use of multiple sequences (T1C, T2, Flair, and T1), which are partitioned into individual voxels namely supervised, unsupervised, and semi-supervised.

For unsupervised models, a set of clusters are generated by determining the resemblance based on the angular inconsistency and feature distances to all voxels [3]. Markov random field (MRF) is a semi-supervised technique used to reduce the issues comprising the overlapping regions and noisy voxel [4]. Multilayer MRF technique is used to detect the existence of BT. It concentrates on distinct information relevant to the intensities. A spatial accuracy weighted hidden MRF (HMRF) and expectation maximization (EM) techniques find useful for resolving the anisotropic problems generated by distinct resolutions of MRI [5]. The conditional random fields (CRF) technique can also be used to detect BT with minimal computation time [6]. Followed by, convolutional neural network (CNN) models find useful as a supervised classification model in which distinct data undergo segmentation without requiring distributed parametric hypothesis [7].

Numerous CNN based architectures are developed to detect and classify BT [8]. They are quickly realized from predicting the individual label to densely connected models namely UNet and DeepMedic. The Hyper column technique finds useful for extracting the details from the CNN model [9]. In addition, U-net and its extended models act as an important series of architecture for the segmentation of medicinal images [10]. The Unet technique offers feature mapping in the encoder to decoder models through consecutive concatenation. Distinct techniques are existed to obtain effectual outcome. The presented model has attained better performance with respect to distinct dataset.

This paper designs an optimal Tsallis entropy based segmentation with deep extreme learning machine (OTSE-DELM) model for medical image classification using MRI images. The presented model involves multilevel thresholding based image segmentation using Tsallis entropy and the optimal threshold values are chosen by black widow optimization (BWO) algorithm. Moreover, the presented model encompasses directional local extrema patterns (DLEP) based feature extractor. Also, the OTSE-DELM model involves DELM based classification model to allocate the proper class labels of the test MRI images. A widespread range of simulations was accomplished to showcase the betterment of the OTSE-DELM model and the outcomes are investigated under distinct dimensions.

\section{The Proposed OTSE-DELM model}

The overall working procedure involved in the OTSE-DELM method is showcased in Fig. 1. The figure stated that the input MRI images are initially preprocessed in two stages namely contrast enhancement and skull stripping. Afterward, the OTSE model is applied to segment the healthy and affected brain regions from the MRI images. Then, the DLEP based feature extraction process is performed to derive a useful set of feature vectors. At last, DELM based classification process gets executed to determine the appropriate class labels.

\subsection{Preprocessing}

Initially, preprocessing is carried out in two levels, namely contrast limited adaptive histogram equalization (CLAHE) [11] and skull stripping. Primarily, CLAHE technique is applied to increase the contrast level of the input MRI images. Then, skull stripping is an important task to segment the MRI images for eradicating the skull regions from the background regions from the MRI for segmentation. It is usually executed by an imaging filter which separates the skull and the residual areas of the images which undergo masking with similarity levels. On the test MRI, normally, the skull or brain areas encompass an increased threshold value (threshold $>200$ ) over the tumor and extra brain regions. So, the image filter is applied to separate the brain regions by the use of a chosen threshold value. Afterward, the skull is removed from the MRI by the use of solidity properties.

\subsection{OTSE based Image Segmentation Process}

Next to the skull stripping process, the OTSE technique is applied to segment the MRI images. Generally, entropy is linked to a metric of disorder in the method. However, Shannon modified the theory of entropy to a measure of uncertainties based on the data content of the scheme. It can be additionally ensured that the Shannon entropy holds the additive property as defined below.

$$
S(X+Y)=S(X)+S(Y)
$$

Using the ideas of multiple fractals, the Tsallis entropy is outspread to non-extensive model utilizing a common entropy form:

$$
S_{q}=\frac{1-\sum_{i=1}^{k}\left(p_{i}\right)^{q}}{q-1}
$$

where $k$ is the number of probabilities of the system and $q$ indicates the degree of non-extensively of the method named Tsallis parameter or entropic index. 


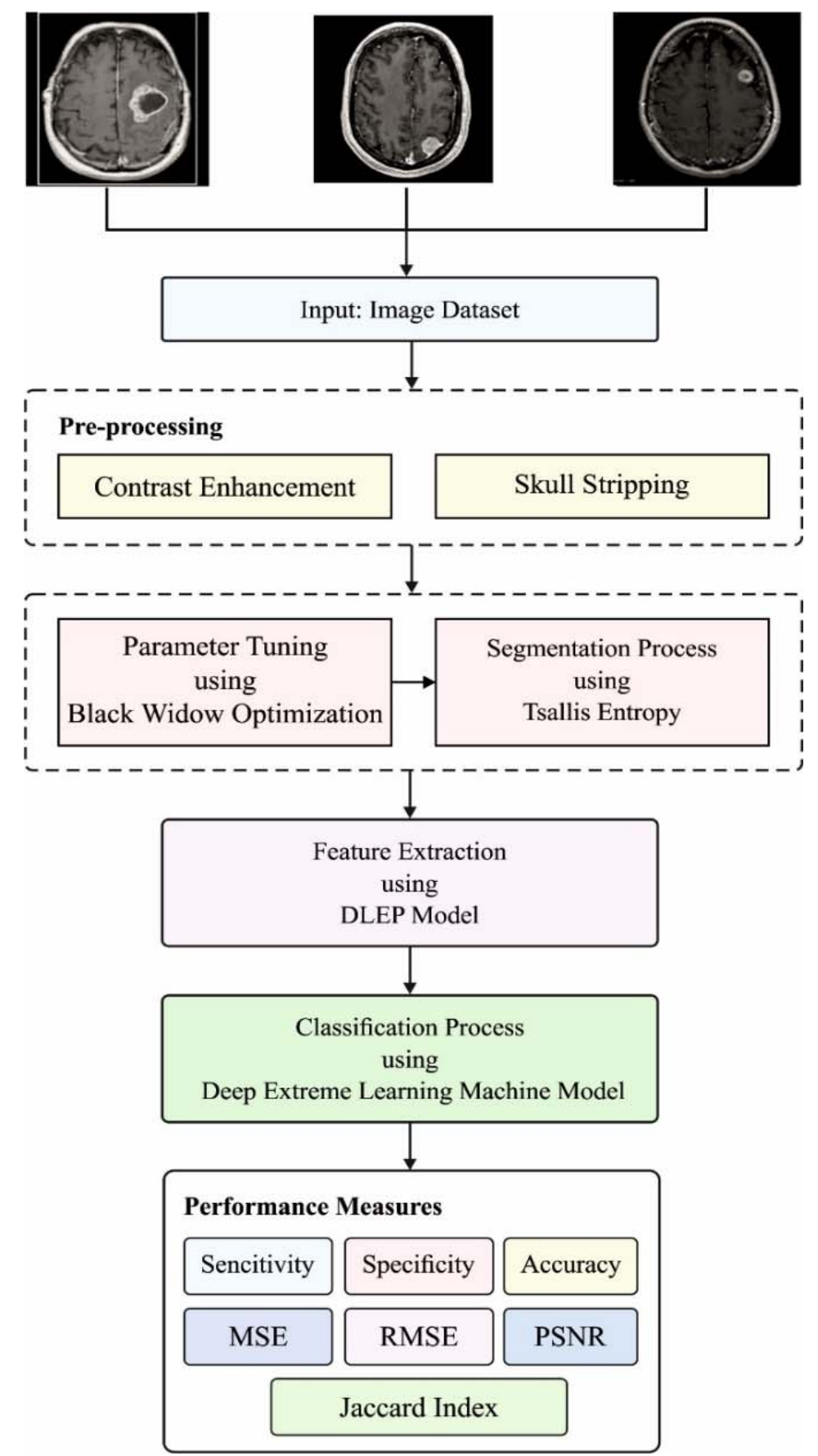

Fig. 1. Working process of OTSE-DELM model.

The entropic system is modified to a statistically independent model using a pseudo-additive entropic rule:

$$
S_{q}(X+Y)=S_{q}(X)+S_{q}(Y)+(1-q) \cdot S_{q}(X) \cdot S_{q}(Y)
$$

This concept is presently utilized for image thresholding. Consider grayscale levels $G$ in the input image and they exist in the interval of $\{1,2 \ldots G\}$. Assume $p_{i}=p_{1}, p_{2} \ldots p_{G}$ be the probability distribution of the grayscale values. Among the two probability distributions, a forefront and backdrop are computed and the probability distribution of these classes is defined below.

where $P^{X}=\sum_{i=1}^{t} p_{i}$ and $P^{Y}=\sum_{i=t+1}^{G} p_{i}$

$$
p_{X}=\frac{p_{1}}{P^{X}}, \frac{p_{2}}{P^{X}}, \ldots, \frac{p_{t}}{F^{X}} \text { and } p_{Y} \frac{p_{t+1}}{P^{Y}}, \frac{p_{t+2}}{P^{Y}}, \ldots, \frac{p_{G}}{P^{Y}}
$$

Then, the Tsallis entropy for all the classes is represented by:

$$
S_{q}^{X}(t)=\frac{1-\sum_{i=1}^{t}\left(p_{i} / P^{X}\right)^{q}}{q-1}
$$




$$
S_{q}^{Y}(t)=\frac{1-\sum_{i=t+1}^{G}\left(p_{i} / P^{Y}\right)^{q}}{q-1}
$$

The information measure amongst the two classes (forefront and backdrop) undergo maximization and the respective grayscale level is treated as the optimal threshold value [12]. It can be attained by the maximization of the objective function involved for bi-level thresholding:

$$
T_{\mathrm{opt}}=\arg \max \left[S_{q}^{X}(t)+S_{q}^{Y}(t)+(1-q) \cdot S_{q}^{X}(t) \cdot S_{q}^{Y}(t)\right]
$$

Subjected to the subsequent limitations of:

$$
\left|P^{X}+P^{Y}\right|-1<S<1-\left|P^{X}-P^{Y}\right|
$$

where

$$
S(t)=S=\left[S_{q}^{X}(t)+S_{q}^{Y}(t)+(1-q) \cdot S_{q}^{X}(t) \cdot S_{q}^{Y}(t)\right]
$$

It can be notable that the constraints are ensured in the presented technique for offering reliability. An optimum threshold value ' $T$ ' is the gray level used for maximizing the Eq. (7) subjected to the constraints of Eq. (8). It is simply defined as multi-level thresholding. The optimum multilevel thresholding issue is represented as an $\mathrm{m}$ dimensional optimization issue. Fascinatingly, ' $m$ ' optimum threshold values $\left[T_{1}, T_{2}, \ldots, T_{m}\right]$ for a provided image is computed for the maximization of the objective function:

$$
\begin{gathered}
{\left[T_{1}, T_{2}, \ldots, T_{m}\right]} \\
=\arg \max \left[S_{q}^{1}(t)+S_{q}^{2}(t)+\cdots+S_{q}^{M}(t)+(1-q) \cdot S_{q}^{1}(t) \cdot S_{q}^{2}(t) \ldots S_{q}^{M}(t)\right](9)
\end{gathered}
$$

where

$$
\begin{aligned}
& S_{q}^{1}(t)=\frac{1-\sum_{i=1}^{t_{1}}\left(p_{i} / P^{1}\right)^{q}}{q-1} \\
& S_{q}^{2}(t)=\frac{1-\sum_{i=t_{1}+1}^{t_{2}}\left(p_{i} / P^{2}\right)^{q}}{q-1}
\end{aligned}
$$

and

$$
S_{q}^{M}(t)=\frac{1-\sum_{i=t_{m}+1}^{G}\left(p_{i} / P^{M}\right)^{q}}{q-1}, M=m+1
$$

Subjected to the collection of constraints represented as follows:

$$
\begin{gathered}
\left|P^{1}+P^{2}\right|-1<S^{1}<1-\left|P^{1}-P^{2}\right| \\
\left|P^{2}+P^{3}\right|-1<S^{2}<1-\left|P^{2}-P^{3}\right| \\
\left|P^{\mathrm{m}}+P^{\mathrm{m}+1}\right|-1<S^{M}<1-\left|P^{\mathrm{m}}-P^{\mathrm{m}+1}\right|
\end{gathered}
$$

were, $P^{1}, P^{2}, \ldots, P^{m+1}$ corresponds to $S^{1}, S^{2}, \ldots, S^{M}$ are determined with $T_{1}, T_{2}, \ldots, T_{m}$, correspondingly. Specifically, the intention is the optimization of the objective function represented in Eq. (9) subjected to the constraints provided in Eq. (10) using BWO algorithm.

To elect optimum threshold values, the BWO algorithm is applied. Owing to the operators involved in the BWO, it is treated as an essential part of evolutionary algorithm (EA). The components of BWOA are identical to GA which is a noteworthy EAs. Similar to other EAs, the BWO has few criteria that are simulated from the process of natural evolution like selection, reproduction, and mutation [13].

These criteria make the difference among every individual EA to another one. But the BWO technique is simulated from the mating performance of the black widow spiders. In addition, it has few variations with the generic EA and it offers effectual solutions to complex issues.

The BWO algorithm is stimulated by the natural selection concept of Darwin. It provides quick convergence and eliminates local optima problems. Therefore, the BWO algorithm has the ability to resolve different optimization problems. Moreover, the BWO algorithm maintains the tradeoff among the exploration and exploitation phases. Fig. 2 illustrates the flowchart of BWO technique [14]. The processes involved in the BWO algorithm are given below.

Step 1: Initialization

Here, $N$ represents the widow population in which every individual widow is defined as an array of $1 \times N_{\text {var }}$ denoting the problem solution. The array can be represented as widow $=\left(x_{1}, x_{2}, \ldots, x_{N v a r}\right)$, where $N_{v}$ ar is the dimension of the optimization issue. In addition, $N_{v a r}$ represents the threshold values required to obtain by the technique whereas $x_{i}$ indicates the $i-$ th candidate solution. 
The fitness of the widow is attained by evaluating the fitness function (FF) of $f$ of the widow of the set $\left(x_{1}, x_{2}, \ldots ., x_{N v a r}\right)$. Afterward, fitness $=f($ widow $)$ is defined as fitness $=f\left(x_{1}, x_{2}, \ldots, x_{N v a r}\right)$. Here, the presented model can replace the $f$ with the FF of Tsallis entropy. The optimization procedure starts with the random initialization of a spider population in a matrix size of $N_{p} o p \times N_{v} a r$. Afterward, the pairs of parents are arbitrarily designated for carrying the procreating procedure next to the mating procedure where the male black widow is eaten by the female.

Step 2: Procreate

At this stage, an array named alpha $\alpha$ needs to be generated as long as a widow array with random numbers encompassing. Next to that, the offspring gets by the use of $\alpha$ where $x_{1}$ and $x_{2}$ are parents, $y_{1}$ and $y_{2}$ are offsprings. The crossover outcome can be determined and saved.

Step 3: Cannibalism

$$
y_{1}=\alpha \times x_{1}+(1-\alpha) \times x_{2} \text { and } y_{2}=\alpha \times x_{2}+(1-\alpha) \times x_{1}
$$

The cannibalism task is divided into sexual and sibling cannibalism. It is noticed when the baby spider eats the mother. Next to the use of cannibalism process, the updated population is determined and saved in a variable named pop2.

Step 4: Mutation

The mutation process takes place through the arbitrary choice of the Mutepop number of individuals from the population which can be mutated. A selected individual solution arbitrarily exchanges 2 components of the array. Next to the mutation process, the new population is assessed and saved in the new population called pop3. At last, the updated population is produced as the migration of pop3 and pop2 are arranged to output the optimal widow of threshold values with $N_{v a r}$ dimension.

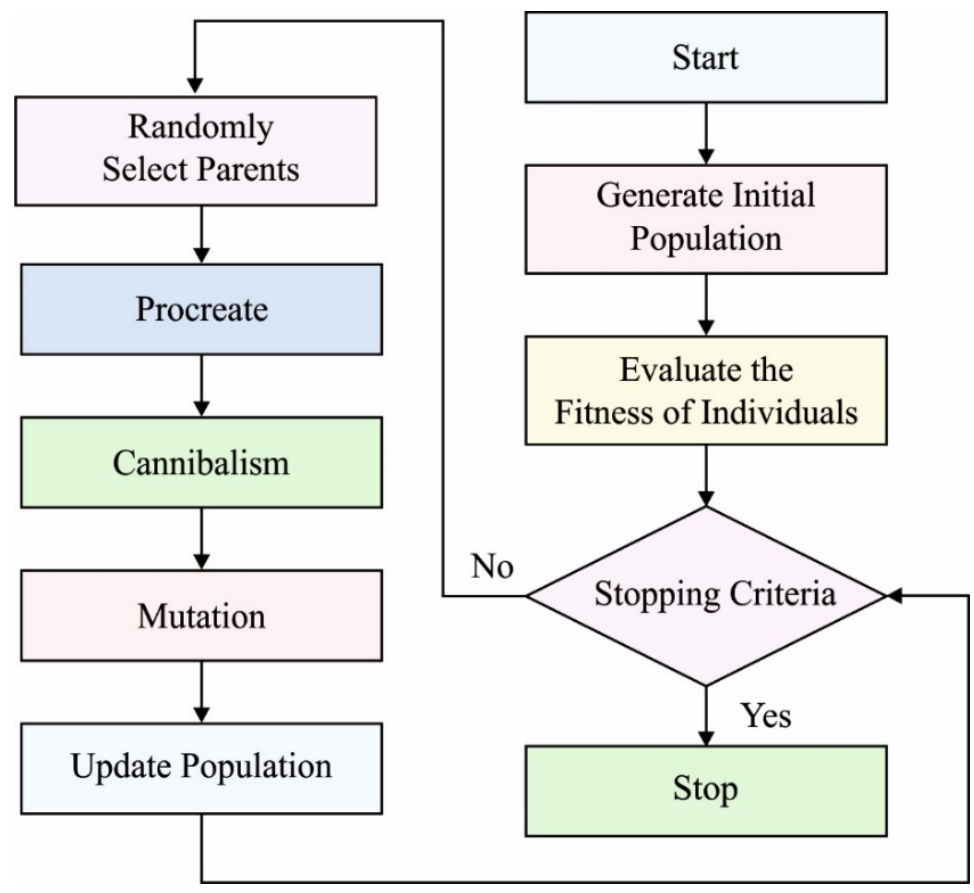

Fig. 2. Flowchart of BWO algorithm.

\subsection{Feature Extraction using DLEP Model}

The segmented MRI image is fed into the DLEP model to generate a useful set of feature vectors. The DLEP model depends on the general idea of Local Binary Pattern (LBP). It is used to define the spatial construction of the local texture by the use of local extrema of middle gray pixel $g_{c}$. For the DLEP on a provided input image, the local extrema in $0^{\circ}, 45^{\circ}, 90^{\circ}$, and $135^{\circ}$ directions are achieved through the computation of local differences amongst the middle and the nearby pixel values as defined in Eq. (12):

$$
I^{\prime}\left(g_{i}\right)=I\left(g_{c}\right)-I\left(g_{i}\right) ; i=1,2, \ldots, 8
$$

The local extrema are defined in Eq. (13).

$$
\begin{aligned}
\hat{I}_{\alpha}\left(g_{c}\right)=f_{3}\left(I^{\prime}\left(g_{j}\right), I^{\prime}\left(g_{j+4}\right)\right) ; j= & (1+\alpha / 45) \\
& \forall \alpha=0^{\circ}, 45^{\circ}, 90^{\circ}, 135^{\circ}
\end{aligned}
$$




$$
f_{3}\left(I^{\prime}\left(g_{j}\right), I^{\prime}\left(g_{j+4}\right)\right)=\left\{\begin{array}{cc}
1 & I^{\prime}\left(g_{j}\right) \times I^{\prime}\left(g_{j+4}\right) \geq 0 \\
0 & \text { else }
\end{array}\right.
$$

The DLEP can be represented $\left(\alpha=0^{\circ}, 45^{\circ}, 90^{\circ}\right.$, and $\left.135^{\circ}\right)$ below:

$$
\left.\operatorname{DLEP}\left(I\left(g_{c}\right)\right)\right|_{\alpha}=\left\{\hat{I}_{\alpha}\left(g_{c}\right) ; \hat{I}_{\alpha}\left(g_{1}\right) ; \hat{I}_{\alpha}\left(g_{2}\right) ; \ldots \hat{I}_{\alpha}\left(g_{8}\right)\right\}
$$

Firstly, the input MRI image is transformed to the DLEP images with the values ranges between 0-511. Next to the computation of DLEP, the whole image is defined by creating the histogram defined in Eq. (16).

$$
\left.H_{D L E P}\right|_{\alpha}(l)=\sum_{j=1}^{N_{1}} \sum_{k=1}^{N_{2}} f_{2}\left(\left.D L E P(j, k)\right|_{\alpha}, l\right) ; l \in[0,511]
$$

where input image size is $N_{1} \times N_{2}$. The DLEP is not identical to the LBP. It will encode the spatial relationship amongst any pair of neighbors in a local region in a predefined direction, whereas the LBP [15] extracts the relationship among the intermediate and adjacent pixels. So, the DLEP will capture extended spatial details over the LBP.

\subsection{DELM based Classification Process}

Finally, DELM model is applied to allocate the class labels to the MRI images using the extracted feature vectors. The ELM model arbitrarily generates the input weights and bias for training and deriving the matrix $\mathrm{H}$, the outcome of the hidden neuron unit. Fig. 3 demonstrates the structure of DELM. Here, the extracted feature vectors are fed to the DELM model to perform classification. The ELM can be represented as:

$$
H \beta=X
$$

where $X$ denotes $N$ labeled data $\left(\mathrm{x}_{i}, \mathrm{t}_{i}\right)$. Based on the ELM, the loss function of deep extreme feature learning can be defined as:

$$
l_{1}=\min _{\beta} \frac{1}{2}\|H \beta-X\|^{2}+\frac{c_{1}}{2}\|H \beta\|^{2}+\frac{c_{2}}{2}\|\beta\|^{2}
$$

where $c_{1}$ and $c_{2}$ represents the smoothness and regularization variables. For optimizing the issue in Eq. (18), the derivative of $\beta$ is determined based on $l_{1}$. For the $k$ th layer of feature learning, the output weight can be defined by:

$$
\beta^{k}=\left(\mathrm{H}_{k}^{T}\left(\left(c_{1}+1\right) \cdot \mathrm{I}_{1}\right) \mathrm{H}_{k}+c_{2} \mathrm{I}_{2}\right)^{-1} \mathrm{H}_{k}^{T} \mathrm{X}^{k-1}
$$

where $\mathrm{I}_{1} \in \Re^{N \times N}$ and $\mathrm{I}_{2} \in \Re^{\widetilde{N} \times \widetilde{N}}$ are identity matrix ( $\widetilde{N}$ is the number of hidden neurons), and $\mathrm{X}^{k-1}$ is the outcome of the $(k-1)$ th layer. When the activation function is $g(x)$, afterward, the outcome of the $k$ th layer is provided as [16]:

$$
\mathrm{X}^{k}=g\left(X^{k-1} \cdot\left(\beta^{k}\right)^{T}\right)
$$

The training process intends to determine the optimum classifier function through the optimization of $\beta$ :

$$
\min _{\beta} \frac{1}{2}\|\mathrm{~F}-\mathrm{T}\|^{2}+\frac{c_{2}}{2}\|\beta\|^{2}
$$

where $\mathrm{F}=H \beta$ and the next term $\frac{c}{2}\|\beta\|^{2}$ norm regularization is appended for guaranteeing the generalization capability, and $c$ is a balance factor. Along with the $\ell_{2,1}$ norm regularization, the Laplacian regularization is included for providing a closed form solution for optimization issues.

$$
\min _{\beta} \frac{1}{2}\|\mathrm{~F}-\mathrm{T}\|^{2}+\frac{c_{2}}{2}\|\beta\|^{2}+\lambda \operatorname{Tr}\left(\mathrm{F}^{T} L F\right)
$$

where $\lambda$ is a balance factor used for controlling the impact of manifold regularization. $\operatorname{Tr}($.$) is the trace of matrix$ and $\mathrm{L}$ is Laplacian matrix. If the edge weights are not obviously declared, an effective model for processing $W$ through the Gaussian kernel:

$$
\mathrm{W}_{i j}=\exp \left(-\frac{\left\|x_{i}-x_{j}\right\|^{2}}{2 \sigma^{2}}\right)
$$

As $\mathrm{F}=H \beta$, the ultimate goal of the classification model is represented as

$$
l_{2}=\min _{\beta}\|J \mathrm{H} \beta-\mathrm{T}\|^{2}+c\|\beta\|^{2}+\lambda \operatorname{Tr}\left((H \beta)^{T} L(H \beta)\right)
$$

where $J=\operatorname{diag}(1, \ldots, 1) \in \mathfrak{R}^{N \times N}$, and $\mathrm{T} \in \mathfrak{R}^{N \times m}$ is the equivalent class labels. The optimal solution of $\beta$ can be defined as

$$
\frac{\partial l_{2}}{\partial \beta}=0 \rightarrow \beta=\left(c \mathrm{I}+\mathrm{H}^{T}(J+\lambda L) H\right)^{-1} \mathrm{H}^{T} \mathrm{JT} .
$$




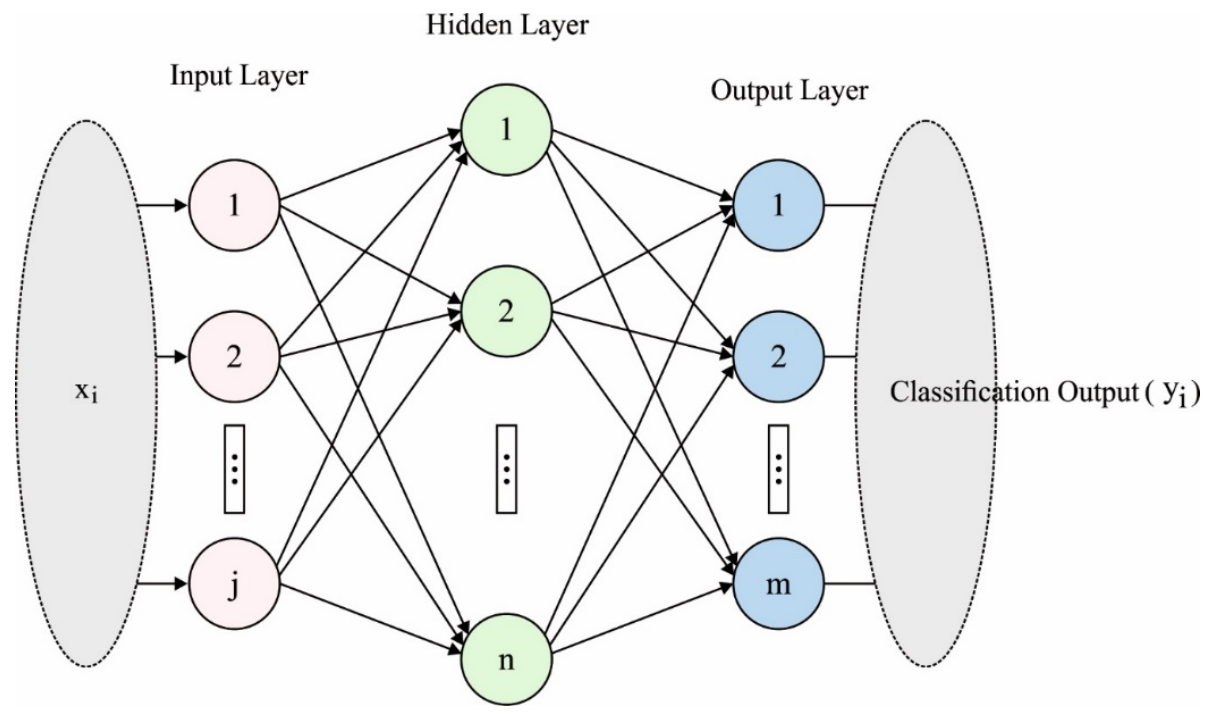

Fig. 3. Structure of DELM.

\section{Performance Validation}

The performance of the OTSE-DELM method has been validated utilizing a benchmark BT MRI image dataset [17]. Fig. 4 depicts the visualization of sample results obtained by the presented OTSE-DELM Model. Fig. 4a depicts the original MRI image, the contrast improved, skull removed, and segmented images are illustrated in Figs. 4b-4d respectively.
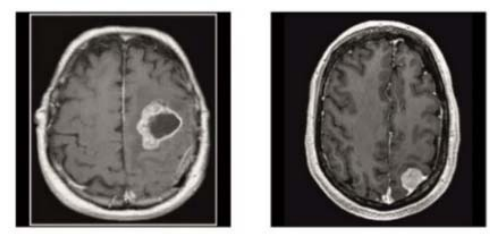

(a)
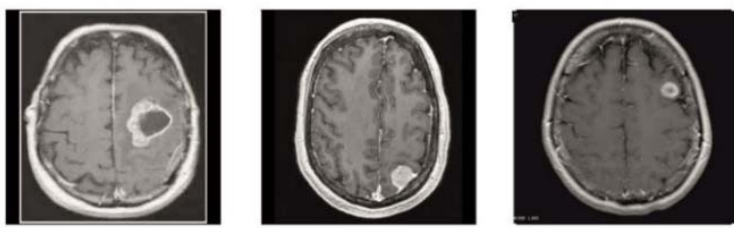

(b)
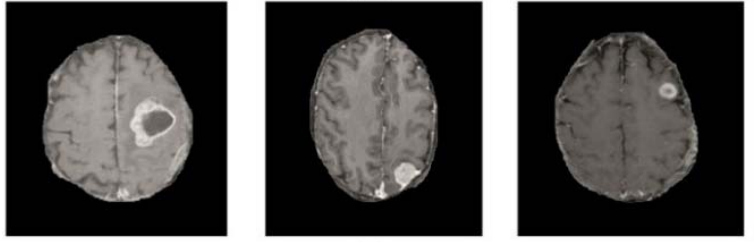

(c)
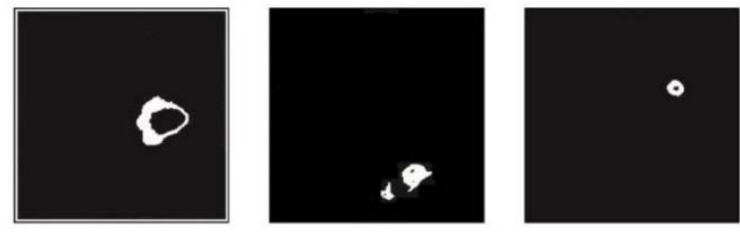

(d)

Fig. 4. a) Original Image b) Contrast Enhanced c) Skull Removal Image c) Segmented Image.

Table 1 and Figs. 5-8 investigates the segmentation outcome of the OTSE model on distinct images. The presented OTSE model has accomplished effectual segmentation performance on all the applied test images. For instance, on the applied image 1, the OTSE model has achieved an MSE of 0.062, RMSE of 0.248, PSNR of 60.239, JI of 0.865 , and accuracy of 97.294. Also, on the applied image 2, the OTSE method has attained an MSE of 0.059, 
RMSE of 0.244 , PSNR of 60.396 , JI of 0.853 , and accuracy of 98.413. Besides, on the applied image 3, the OTSE technique has reached an MSE of 0.057, RMSE of 0.239, PSNR of 60.569, JI of 0.869, and accuracy of 98.650. Moreover, on the applied image 4, the OTSE approach has attained an MSE of 0.052, RMSE of 0.228, PSNR of 60.963 , JI of 0.859 , and accuracy of 97.420 . Furthermore, on the applied image 5, the OTSE methodology has reached an MSE of 0.057 , RMSE of 0.239 , PSNR of 60.558 , JI of 0.864 , and accuracy of 97.927 . Eventually, on the applied image 6, the OTSE approach has achieved an MSE of 0.059 , RMSE of 0.243 , PSNR of 60.430 , JI of 0.872 , and accuracy of 97.050. In line with, on the applied image 7, the OTSE technique has reached an MSE of 0.052 , RMSE of 0.227 , PSNR of 61.000 , JI of 0.861 , and accuracy of 98.300 . Meanwhile, on the applied image 8, the OTSE methodology has attained an MSE of 0.059, RMSE of 0.243 , PSNR of 60.435 , JI of 0.867 , and accuracy of 97.797. Concurrently, on the applied image 9, the OTSE methodology has attained an MSE of 0.063, RMSE of 0.251 , PSNR of 60.122 , JI of 0.870 , and accuracy of 98.050. Simultaneously, on the applied image 10, the OTSE technique has obtained an MSE of 0.055, RMSE of 0.235 , PSNR of 60.702 , JI of 0.869 , and accuracy of 98.190 .

Table 1. Result Analysis of Proposed OTSE Based Segmentation Method in terms of Different Measures.

\begin{tabular}{|l|l|l|l|l|l|}
\hline No. of Images & MSE & RMSE & PSNR & JI & Accuracy \\
\hline Image 1 & 0.062 & 0.248 & 60.239 & 0.865 & 97.294 \\
\hline Image 2 & 0.059 & 0.244 & 60.396 & 0.853 & 98.413 \\
\hline Image 3 & 0.057 & 0.239 & 60.569 & 0.869 & 98.650 \\
\hline Image 4 & 0.052 & 0.228 & 60.963 & 0.859 & 97.420 \\
\hline Image 5 & 0.057 & 0.239 & 60.558 & 0.864 & 97.927 \\
\hline Image 6 & 0.059 & 0.243 & 60.430 & 0.872 & 97.050 \\
\hline Image 7 & 0.052 & 0.227 & 61.000 & 0.861 & 98.300 \\
\hline Image 8 & 0.059 & 0.243 & 60.435 & 0.867 & 97.797 \\
\hline Image 9 & 0.063 & 0.251 & 60.122 & 0.870 & 98.050 \\
\hline Image 10 & 0.055 & 0.235 & 60.702 & 0.869 & 98.190 \\
\hline Average & $\mathbf{0 . 0 5 8}$ & $\mathbf{0 . 2 4 0}$ & $\mathbf{6 0 . 5 4 1}$ & $\mathbf{0 . 8 6 5}$ & $\mathbf{9 7 . 9 0 9}$ \\
\hline
\end{tabular}

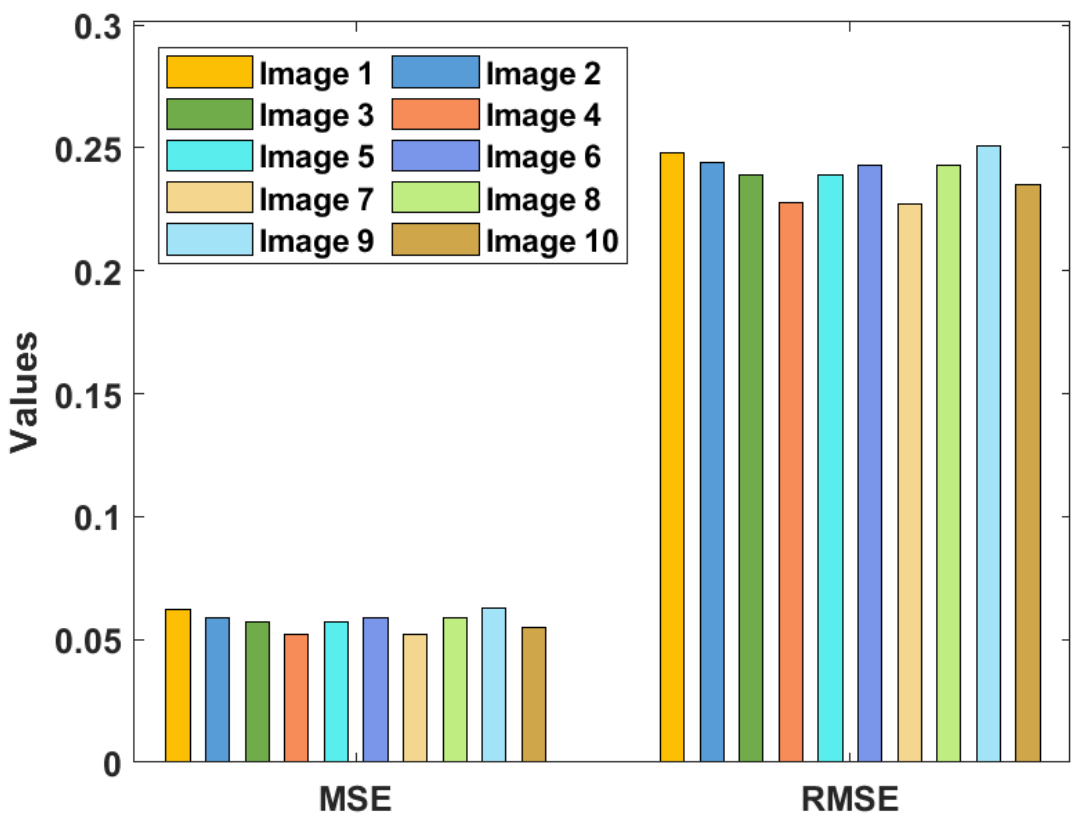

Fig. 5. MSE and RMSE analysis of OTSE model. 


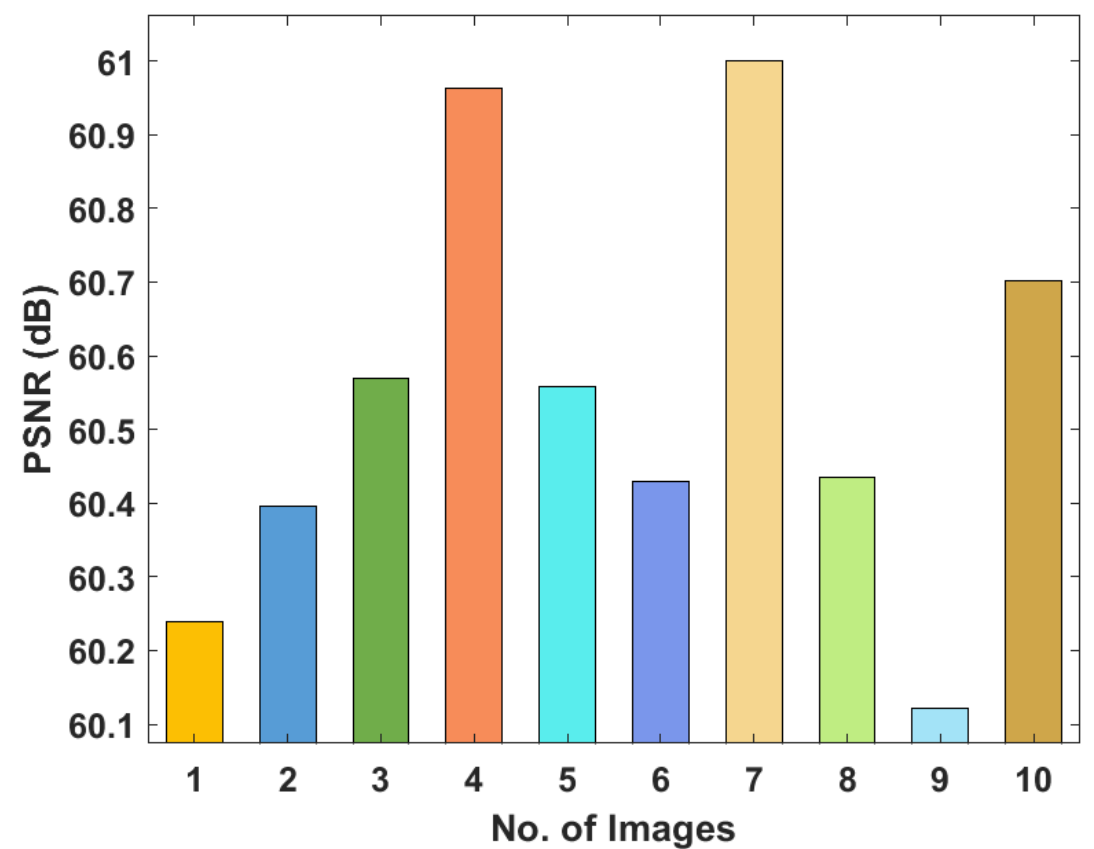

Fig. 6. PSNR analysis of OTSE model.

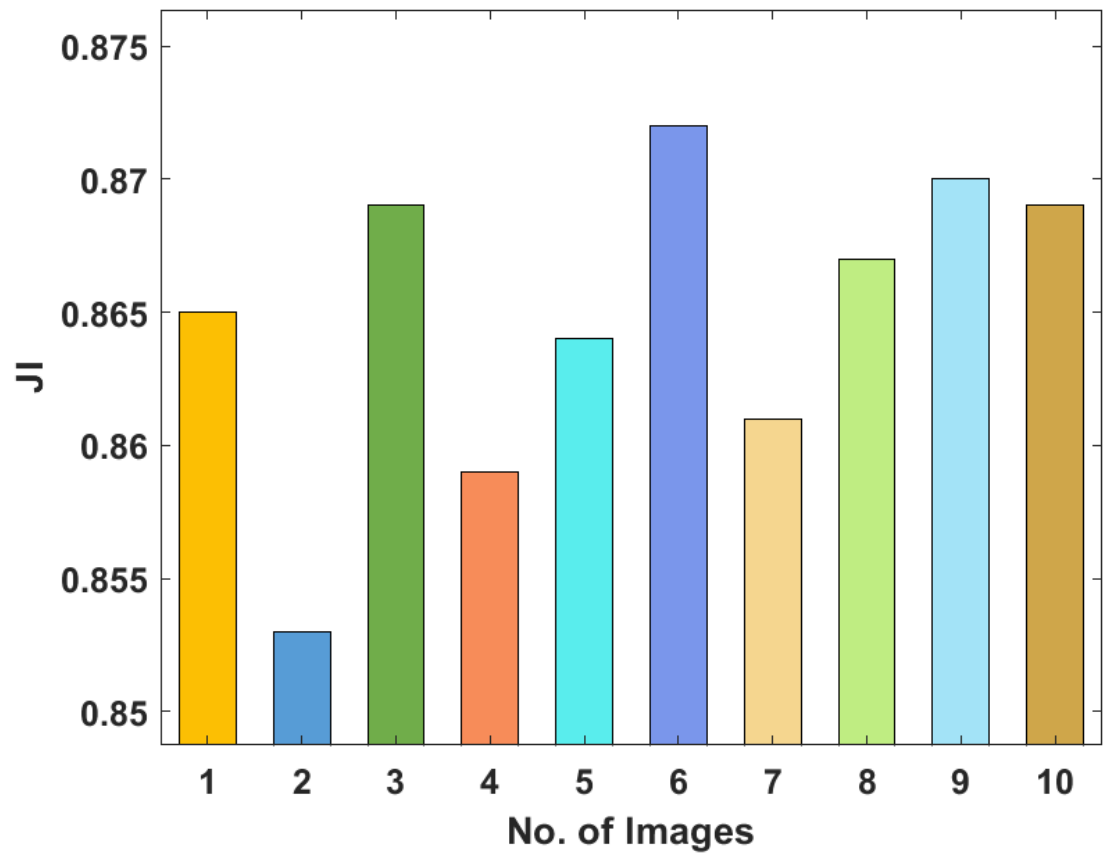

Fig. 7. JI analysis of OTSE model. 


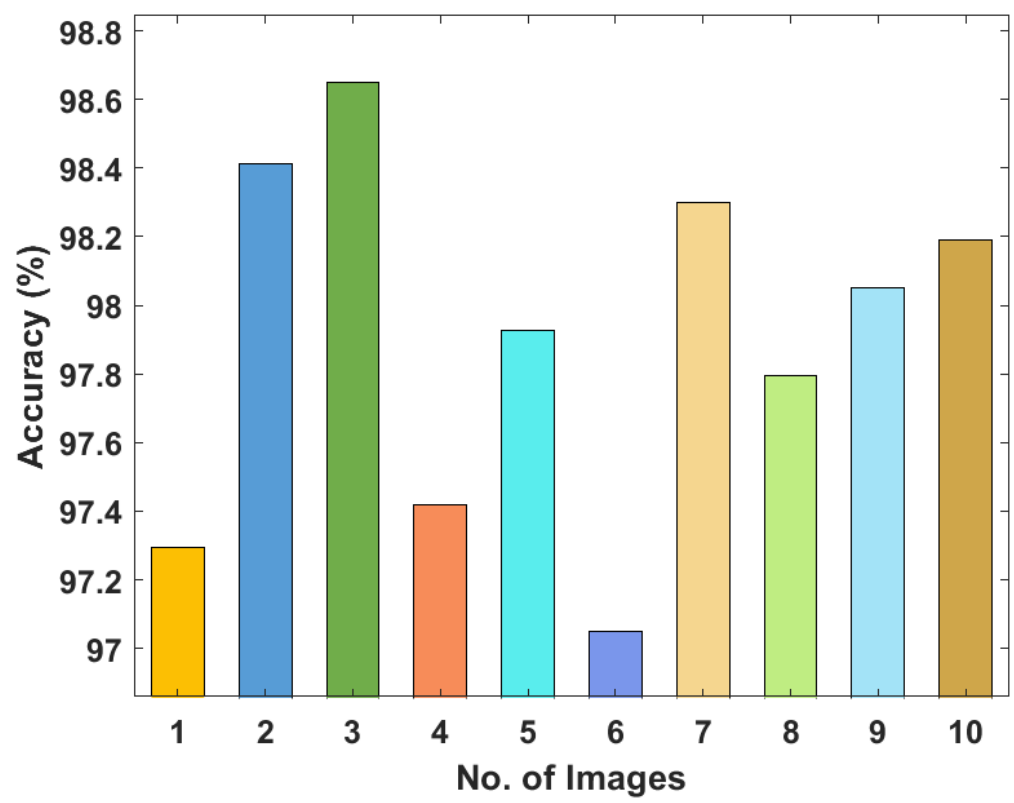

Fig. 8. Accuracy analysis of OTSE model.

Table 2 and Figs. 9-10 examines the segmentation results analysis of the OTSE with TLBO based multi-level thresholding techniques interms of PSNR and JI [18]. From the results, it is evident that the TLBO-Tsallis method has accomplished inferior segmentation outcomes with the PSNR of $15.321 \mathrm{~dB}$ and 0.742 . On continuing with, the TLBO-Kapur's method has also obtained closer PSNR of $15.718 \mathrm{~dB}$ and JI of 0.771 . At the same time, it is noticed that the TLBO-Shannon model has accomplished resole segmentation with the PSNR of 26.340dB and JI of 0.837 . But the presented OTSE model has shown its betterment in the segmentation process with the maximum PSNR of $60.541 \mathrm{~dB}$ and JI of 0.865 .

Table 2. Segmentation Results of Proposed OTSE with recent technique with respect to PSNR and JI.

\begin{tabular}{|l|l|l|}
\hline Methods & PSNR (dB) & JI \\
\hline Proposed OTSE & 60.541 & 0.865 \\
\hline TLBO-Kapurs & 15.718 & 0.771 \\
\hline TLBO-Tsallis & 15.321 & 0.742 \\
\hline TLBO-Shannon & 26.340 & 0.837 \\
\hline
\end{tabular}

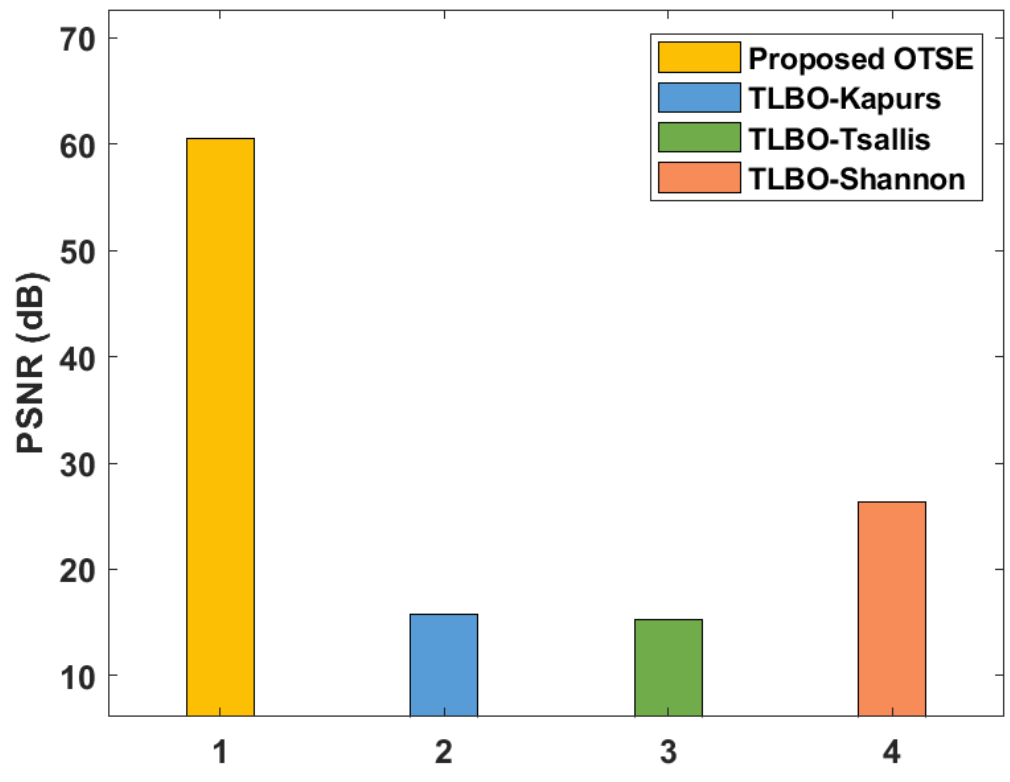

Fig. 9. Result analysis of OTSE model interms of PSNR. 


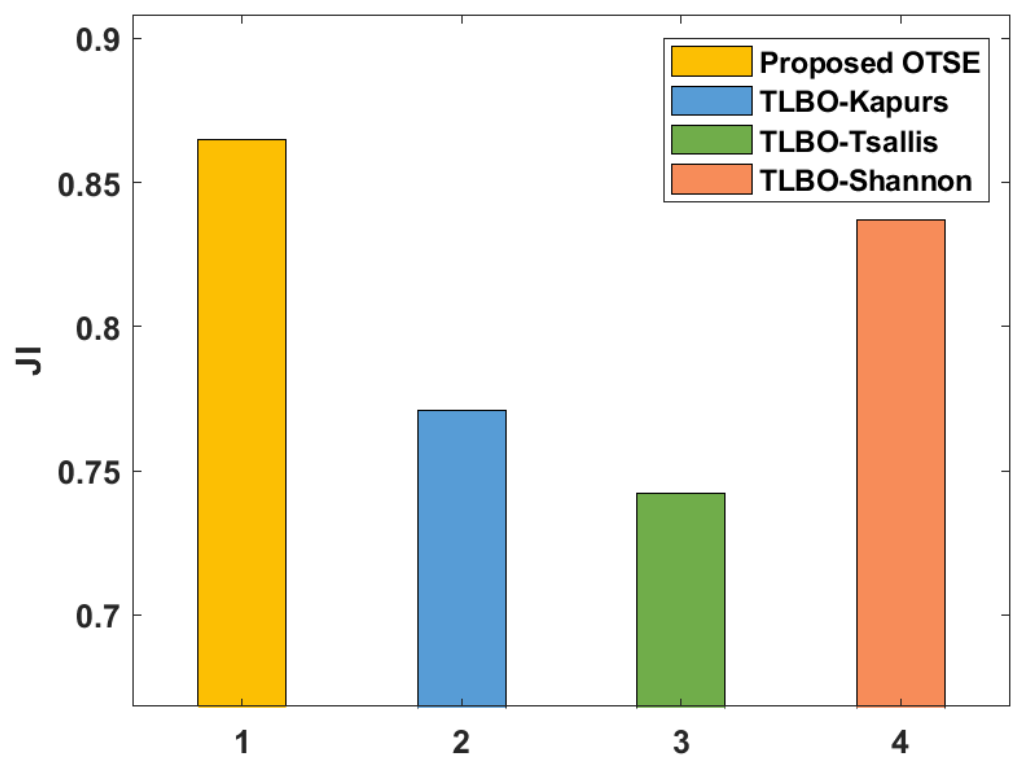

Fig. 10. Result analysis of OTSE model interms of JI.

Table 3 and Fig. 11 give a comparative classification outcomes analysis of the OKE-ANFIS method with respect to different measures $[19,20]$. From the experimental result, it can be apparent that the CNN method has failed to show optimal classification with the sensitivity, specificity, and accuracy of $91.2 \%, 93.4 \%$, and $93.3 \%$ respectively. In line with, the MM-DCNN technique has achieved somewhat improved performance by offering a sensitivity, specificity, and accuracy of $92.6 \%, 93 \%$, and $93.3 \%$. Also, the AS-CNN approach has tried to demonstrate moderate results by achieving a sensitivity, specificity, and accuracy of $94.2 \%, 94.4 \%$, and $94.6 \%$ correspondingly. Likewise, the Modified AdaBoost model has exhibited slightly acceptable results with sensitivity, specificity, and accuracy of $94.3 \%, 95.1 \%$, and $95.9 \%$ respectively. Concurrently, the ANFIS method has resulted in a reasonable performance with the sensitivity, specificity, and accuracy of $96.2 \%, 95.1 \%$, and $96.4 \%$. Followed by, a competitive classification performance with the sensitivity, specificity, and accuracy of $97.17 \%, 98.77 \%$, and $98.07 \%$ have been offered by the D-CNN method. But, the OTSE-DELM approach has showcased effectual classification results with superior sensitivity, specificity, and accuracy of $98.34 \%, 98.98 \%$, and $98.76 \%$.

Table 3. Comparative studies of presented OTSE-DELM with recent models on Classification Results.

\begin{tabular}{|l|l|l|l|}
\hline Methods & Sensitivity & Specificity & Accuracy \\
\hline OTSE-DELM & 98.34 & 98.98 & 98.76 \\
\hline D-CNN & 97.17 & 98.77 & 98.07 \\
\hline ANFIS & 96.20 & 95.10 & 96.40 \\
\hline CNN & 91.20 & 93.40 & 93.30 \\
\hline AS-CNN & 94.20 & 94.40 & 94.60 \\
\hline MM-DCNN & 92.60 & 93.00 & 93.30 \\
\hline Modified AdaBoost & 94.30 & 95.10 & 95.90 \\
\hline
\end{tabular}




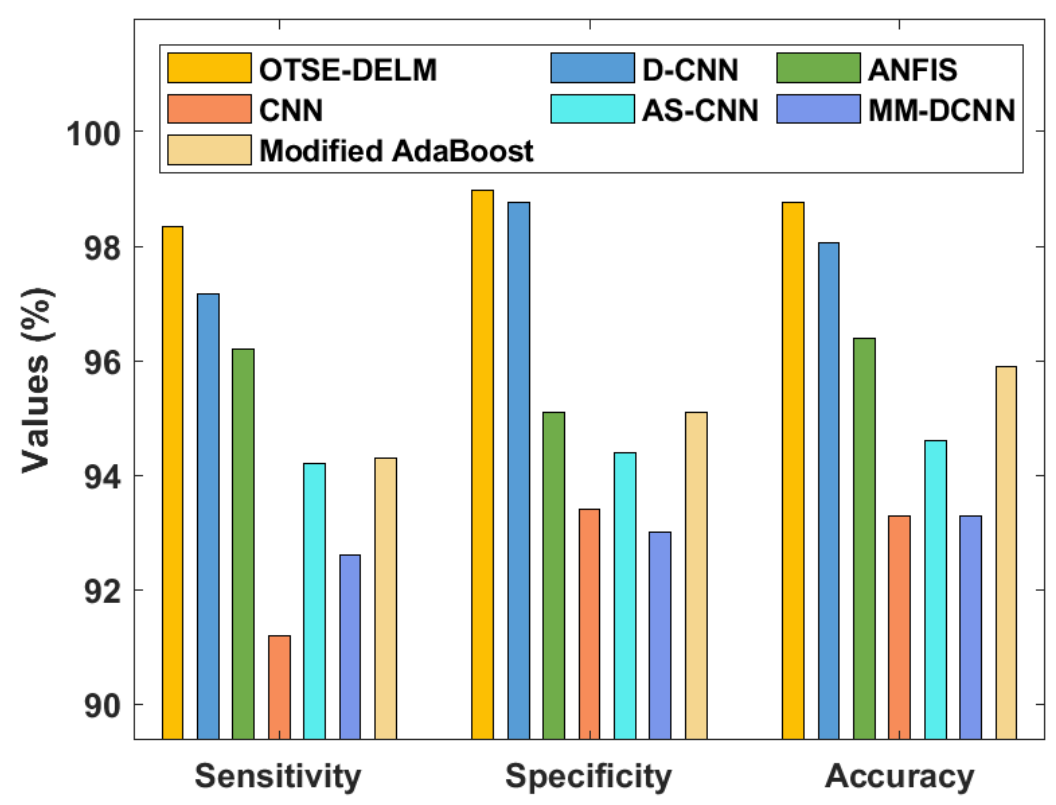

Fig. 11. Comparative analysis of OTSE-DELM model with different measures.

\section{Conclusion}

This paper has designed a novel OTSE-DELM model for medical image classification using MRI images. Primarily, the input MRI images are initially preprocessed to strip the skull. Afterward, the OTSE model is applied to segment the healthy and affected brain regions from the MRI images. The presented model involves multi-level thresholding based image segmentation using Tsallis entropy and optimal threshold values are chosen by BWO algorithm. Then, the DLEP based feature extraction process is performed to derive a useful set of feature vectors. At last, DELM based classification process gets executed to determine the appropriate class labels. A wide range of simulations was performed to showcase the betterment of the OTSE-DELM method and the outcomes are investigated under distinct dimensions. The experimental values indicated that the OTSE-DELM model has obtained improved classification results with the sensitivity of $98.34 \%$, specificity of $98.98 \%$, and accuracy of $98.76 \%$.

\section{References}

[1] Amin, J., Sharif, M., Yasmin, M., and Fernandes, S.L., A distinctive approach in brain tumor detection and classification using MRI. Pattern Recognition Letters, 2017.

[2] Zhang, L., Song, M., Liu, X., Bu, J., and Chen, C.J.S.P., Fast multiview segment graph kernel for object classification. 93 (6):1597$1607,2013$.

[3] Zhang, L., Han, Y., Yang, Y., Song, M., Yan, S., and Tian, QJIToIP., Discovering discriminative graphlets for aerial image categories recognition. 22 (12):5071-5084, 2013.

[4] Menze, B.H., Van Leemput, K., Lashkari, D., Weber, M.-A., Ayache, N., and Golland, P., A generative model for brain tumor segmentation in multi-modal images. International conference on medical image computing and computer-assisted intervention, Springer. 151-159, 2010.

[5] Cheng, G., Zhou, P., and Han, JJIToIP., Duplex metric learning for image set classification. 27 (1):281-292, 2018.

[6] Lee, C.-H., Wang, S., Murtha, A., Brown, M.R., and Greiner, R., Segmenting brain tumors using pseudo-conditional random fields. International conference on medical image computing and computer-assisted intervention. Springer. 359-366, 2008.

[7] Zhang, C., Fang, M., and Nie, H., Brain tumor segmentation using fully convolutional networks from magnetic resonance imaging. J. Med. Imag. Health Inform. 8(8):1546-1553, 2018.

[8] Amin, J., Sharif, M., Yasmin, M., and Fernandes, S. L., Big data analysis for brain tumor detection: Deep convolutional neural networks. Fut. Gen. Comput. Syst. 87:290-297, 2018.

[9] Bernal, J., Kushibar, K., Asfaw, D. S., Valverde, S., Oliver, A., Martí, R., and Lladó, X., Deep convolutional neural networks for brain image analysis on magnetic resonance imaging: A review. Artificial intelligence in medicine, 2018.

[10] Isensee, F., Petersen, J., Klein, A., Zimmerer, D., Jaeger, P.F., Kohl, S., Wasserthal, J., Koehler, G., Norajitra, T., and Wirkert, S., Nnunet: Self-adapting framework for u-net-based medical image segmentation. arXiv preprint arXiv:180910486, 2018.

[11] Reza, A.M., 2004. Realization of the contrast limited adaptive histogram equalization (CLAHE) for real-time image enhancement. Journal of VLSI signal processing systems for signal, image and video technology, 38(1), pp.35-44.

[12] Agrawal, S., Panda, R., Bhuyan, S. and Panigrahi, B.K., 2013. Tsallis entropy based optimal multilevel thresholding using cuckoo search algorithm. Swarm and Evolutionary Computation, 11, pp.16-30.

[13] Houssein, E.H., Helmy, B.E.D., Oliva, D., Elngar, A.A. and Shaban, H., 2020. A novel Black Widow Optimization algorithm for multilevel thresholding image segmentation. Expert Systems with Applications, p.114159.

[14] Hayyolalam, V. and Kazem, A.A.P., 2020. Black widow optimization algorithm: A novel meta-heuristic approach for solving engineering optimization problems. Engineering Applications of Artificial Intelligence, 87, p.103249.

[15] Murala, S., Maheshwari, R.P. and Balasubramanian, R., 2012. Directional local extrema patterns: a new descriptor for content based image retrieval. International journal of multimedia information retrieval, 1(3), pp.191-203. 
[16] Khatab, Z.E., Hajihoseini, A. and Ghorashi, S.A., 2017. A fingerprint method for indoor localization using autoencoder based deep extreme learning machine. IEEE sensors letters, 2(1), pp.1-4.

[17] https://www.cancerimagingarchive.net/

[18] Rajinikanth, V., Satapathy, S.C., Fernandes, S.L. and Nachiappan, S., 2017. Entropy based segmentation of tumor from brain MR images-a study with teaching learning based optimization. Pattern Recognition Letters, 94, pp.87-95.

[19] Selvapandian, A. and Manivannan, K., 2018. Fusion based glioma brain tumor detection and segmentation using ANFIS classification. Computer methods and programs in biomedicine, 166, pp.33-38.

[20] Ganesan, M., Sivakumar, N. and Thirumaran, M., 2020. Internet of medical things with cloud-based e-health services for brain tumour detection model using deep convolution neural network. Electronic Government, an International Journal, 16(1-2), pp.69-83. 